\title{
Bayesian Approach for Inverse Problems in Optical Coherent and Non Coherent Imaging
}

\author{
Ali MOHAMmaD-DJAFARI \\ Laboratoire des Signaux et Systèmes (CNRS - SUPÉLEC - UPS). \\ SupÉLEC, Plateau de Moulon, 91192 Gif-sur-Yvette Cedex, France.
}

\begin{abstract}
In many applications of optical imaging or diffraction scattering (ultrasounds or microwave), one of the main mathematical part of the inversion, problems, when linearized, become a Fourier synthesis (FS) one. This problem consists in estimating a multivariable function from the measured data which correspond to partial knowledge of its Fourier transform (FT). ${ }^{1-5}$ Most classical methods of inversion are based on interpolation of the data and fast inverse FT. ${ }^{6}$ But, when the data do not fill uniformly the Fourier domain or when the phase of the signal is lacking as in optical interferometry, the results obtained by such methods are not satisfactory, because these inverse problems are ill-posed. The Bayesian estimation approach, via an appropriate modeling of the unknowns gives the possibility of compensating the lack of information in the data, thus giving satisfactory results. In this paper we give an example of FS problem in an interferometry imaging.
\end{abstract}

\section{INTRODUCTION}

In many applications of optical interferometry or diffraction scattering imaging (ultrasounds or microwave), one of the main mathematical part of the inversion, problems, when linearized, become a Fourier synthesis (FS) one. $^{1-5}$ This problem consists in estimating a multivariable function $f(\boldsymbol{x})$ (often an image) from the measured data which are, either directly or after a transformation, related to the Fourier transform of $f(\boldsymbol{x})$ :

$$
g_{i}(\boldsymbol{\omega})=\mathcal{G}\left(g\left(\boldsymbol{\omega}_{i}\right)\right), i=1, \cdots, M \text { with } g(\boldsymbol{\omega})=\int f(\boldsymbol{x}) \exp \left\{-j \boldsymbol{\omega}^{t} \cdot \boldsymbol{x}\right\} \mathrm{d} \boldsymbol{x}
$$

where $\mathcal{G}($.$) is a known function, for example \mathcal{G}(s)=s$ or the absolute value $\mathcal{G}(s)=|s|$ where $s$ is a complex value quantity.

In some applications, such as X-ray tomography, diffraction tomography or Eddy current tomography, the 1D FT of the measured data give the necessary information in the Fourier domain. In some other applications, such as Nuclear magnetic resonance (NMR), SAR or Radar or interferometric data in radioastronomy, the data are directly the points in the Fourier domain. In some cases such as coherent imaging, we have complex valued data (both magnitude and phase are measured) but in some others, such as in incoherent imaging, only the magnitude can be measured. In all cases, the Fourier domain data are incomplete and also perturbed by the measurement noise.

In $2 \mathrm{D}$ imaging, the mathematical problem becomes the estimation of an image $f(x, y)$ from partial and noisy knowledge of its FT $g(u, v)$ :

$$
g_{i}(u, v)=\mathcal{G}\left(g\left(u_{i}, v_{i}\right)\right), i=1, \cdots, M \text { with } g(u, v)=\iint f(x, y) \exp \{-j(u x+v y)\} \mathrm{d} x \mathrm{~d} y .
$$

The partial knowledge is often related to the support of the data gathering system and the lack of phase measurement. The following figure shows the support of the data gathering in some tomographic imaging systems such as X-rays, NMR, microwave diffraction tomography, ${ }^{7,8}$ SAR and Radar and Eddy current imaging. ${ }^{9}$ 


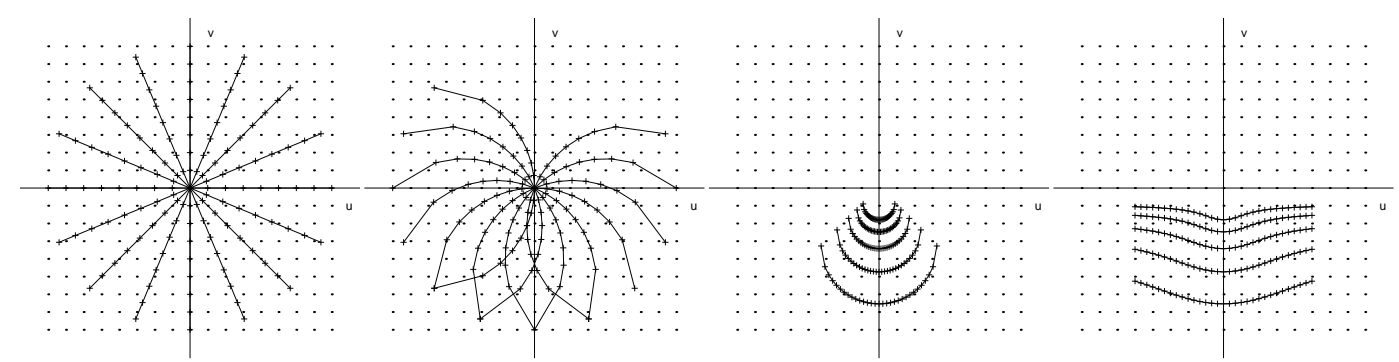

Figure 1. Algebraic contours representing the support of the data in Fourier synthesis problems in different imaging systems: a) X-ray tomography and NMR imaging, b) Diffraction tomography with Born approximation, c) SAR and RADAR imaging, d) Eddy current tomography.

\section{CLASSICAL INTERPOLATION METHODS}

Many classical inversion techniques for such inverse problem consist mainly of interpolation in the Fourier domain to fill the domain on a Cartesian grid and then inverse FT to construct an image as an estimate of the desired solution. ${ }^{10-12}$

These methods, also very fast and used with success in many cases, cannot give satisfactory results when the support of the Fourier domain data are too sparse or when the data are noisy (particularly when the phase measurement is less accurate or missing).

One can also interpret such methods as the optimizer of a least square (LS) criterion

$$
\widehat{f}=\arg \min _{f \in \mathcal{F}}\{Q(f)\} \quad \text { with } Q(f)=\sum_{i}\left(g_{i}-\mathcal{G}\left(g\left(\boldsymbol{\omega}_{i}\right)\right)\right)^{2} .
$$

It is then easy to see that, in these methods, the solution is just the inverse FT of the available data assuming that the unobserved data are equal to zero. Indeed, many interpolation techniques are based on the assumption of the prior knowledge of the support of the unknown function.

A second class of the methods try to optimize a functional $\Omega(f)$ representing some properties of the solution subject to the data constraints Equation [1]. We may mention the Euclidean norms $\|f\|^{2}$ or $\|\nabla f\|^{2}$

$$
\Omega(f)=\|\left.\nabla f\right|^{2}=\int|\nabla f(\boldsymbol{x})|^{2} \mathrm{~d} \boldsymbol{x}
$$

or the entropy

$$
\Omega(f)=\int f(\boldsymbol{x}) \ln f(\boldsymbol{x}) \mathrm{d} \boldsymbol{x}
$$

as two examples.

There are many methods which try to implement these optimization problems. We may mention the Gerchberg-Papoulis ${ }^{13-18}$ or Gerchberg-Saxton iterative type algorithms which use the prior knowledge of the support of the data and sometimes the positivity of the solution to obtain satisfactory results. The main idea in both methods is to go alternatively to the space and the to the Fourier domain and apply the support and positivity constraint and data constraints respectively.

\section{REGULARIZATION METHODS}

The main idea behind the inversion methods developed in our laboratory is to add some prior information to compensate for the missing data. Note however that this prior information must stay very general. In our case we try to use the prior informations such as positivity, global or local continuity, regional homogeneity, binary valued or compact body in a homogeneous background, etc. 
Two main approaches give the necessary tools to account for these kind of information: deterministic regularization theory and probabilistic Bayesian inference and statistical estimation theory. Even if the probabilistic Bayesian inference is more general, there is tight relation between the two approaches. In particular, it is easy to show the Bayesian maximum a posteriori (MAP) estimation approach becomes equivalent to the regularization. In both cases, the solution to the inversion problem is defined as the optimizing value of a compound criterion:

$$
\widehat{f}=\arg \min _{f \in \mathcal{F}}\{Q(f)+\lambda \Omega(f)\} .
$$

with two parts: A data adequation part $Q(f)$

$$
Q(f)=\sum_{i}\left(g_{i}-\mathcal{G}\left(g\left(\boldsymbol{\omega}_{i}\right)\right)\right)^{2} .
$$

where $\boldsymbol{g}=\left\{g_{i}, i=1, \cdots, M\right\}$ represent the observed data; and an a priori part $\Omega(f)$. Very often $Q(f)$ is choosed to be the least square (LS) criterion (7) and different regularization criteria differ mainly in the choice of $\Omega(f)$. For example, the global regularity of the solution is insured by choosing

$$
\Omega(f)=\int \phi(|\nabla f(\boldsymbol{x})|) \mathrm{d} \boldsymbol{x}
$$

where $\nabla f(\boldsymbol{x})$ is the gradient of $f$ and $\phi$ is a monotonically increasing positive function.

The piecewise continuity (or piecewise homogeneity) can be obtained by choosing a non convex function for $\phi$ (for example a truncated quadratic $\phi(t)=t^{2}, \quad \forall|t|<T$ and $\phi(t)=T^{2}-T+|t|, \quad \forall \geq T$ ) or by a $\phi$ function non-derivable at the origin such as $\phi(t)=|t|$.

The positivity can be insured by choosing

$$
\Omega(f)=\int f(\boldsymbol{x}) \ln f(\boldsymbol{x}) \mathrm{d} \boldsymbol{x}
$$

\section{PIECEWISE HOMOGENEOUS MODELING}

An important property in many image reconstruction applications is the local and regional homogeneity of the images, i.e., when we know that the images are homogeneous inside each region, but we do not know neither the shapes nor the positions of those regions. A criterion which can account for this has the following form:

$$
\Omega(f)=\sum_{k=0}^{K} \int_{\boldsymbol{x} \in R_{k}} \frac{1}{\sigma_{k}^{2}}\left(f(\boldsymbol{x})-m_{k}\right)^{2} \mathrm{~d} \boldsymbol{x}
$$

where $R_{k}$ represents the support of the region $k, m_{k}$ the mean value of the pixels in that region, $\sigma_{k}^{2}$ the variance of the values of those pixels and $K$ the number of those regions.

Each region $R_{k}$ can be the union of smaller sub-regions (connex or not)

$$
R_{k}=\cup_{l=0}^{L_{k}} R_{k l} .
$$

and we may want to impose some constraints on the shape of those sub-regions : connexity, compacity, convexity, polygonal or polyhedral, etc. We may also want to impose some constraints on their number and their contour shapes parameters (mean length or mean curvature).

The main difficulty is then the estimation of of the shape of these regions. When this is doe, the estimation of other hyperparameters such as $\left(m_{k}, \sigma_{k}^{2}\right)$, for $k=1, \ldots, K$ become easy.

The case $K=2$ is an interesting one which is encountered in many non-destructive testing (NDT) application where one looks for a default region $(k=1)$ inside a homogeneous background $(k=0)$. This case can easily extended to $K=3$ where, for example, $k=0$ corresponds to the background region, $k=1$ to the inner side of a moderately default region and $k=2$ to the inner side of a hard default region.

We are developing methods for inverse problems in general for this kind of situations. In the following, we will see that the Bayesian estimation approach is a more appropriate approach for handling this kind of modeling via an appropriate a priori probability density function. 

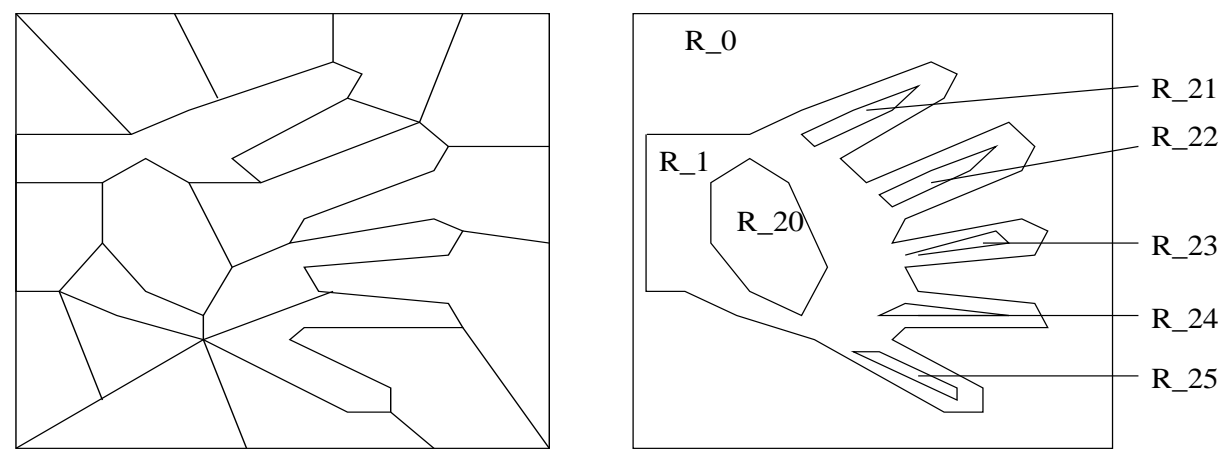

Figure 2. Piecewise homogeneous regions and hierarchically embedded compact homogeneous regions

\section{BAYESIAN APPROACH AND ITS COMPUTATIONAL ASPECTS}

The main idea behind the Bayesian estimation approach is to write down all the prior information on the problem in two prior s, $p(g \mid f)$ and $p(f)$ and then deduce the posterior probability law $p(f \mid g)$, from which we can make any inference about the unknown $f$. The first prior law $p(g \mid f)$ accounts for all the modeling errors and $p(f)$ for all the prior knowledge on the unknown $f$ which is needed to complete the information content of the data on $f$ which is translated in $p(g \mid f$. Thus, the posterior probability law $p(f \mid g)$ combines optimally both of them. Noting that $p(f \mid g) \propto p(g \mid f) p(f)$, if we choose as a point estimator the Maximum a posteriori (MAP) estimate: , or equivalently $\widehat{f}=\arg \min _{f}\{-\ln p(f \mid g)=-\ln p(g \mid f)-\ln p(f)\}$, we see that there exist a link between the Bayesian MAP estimation approach and the regularization approach, because we can always assume $Q(f)=-\ln p(g \mid f)$ as a kind of data adequation measure and $\Omega(f)=-\ln p(f)$ as a regularization functional. However, the Bayesian approach has many other advantages and tools (expectation, marginalization, variance and covariance) which becomes a more appropriate approach to handle with inverse problems. However, even if it is possible to deal with continuous functions and integral equations (infinite dimensions), it is easier to interpret the probability laws in a discretized version of integral equations (finite dimensions). But anyway, to do numerical computation, we need to discretize the integral equations.

\subsection{Discretization}

The first step for developing any computational algorithm for inverse problems is the discretization of the integral equation of the forward problem. In the case of Fourier synthesis problem, if we assume that the space is discretized in pixels with unit dimensions, then we can write

$$
g(u, v)=\sum_{x} \sum_{y} f(x, y) \exp \{j(u x+v y)\}
$$

Then, noting by $g\left(u_{i}, v_{i}\right)=g_{i}, i=1, \cdots, M$ the gathered data and putting all the pixel values $f(x, y), x=$ $1, \cdot, N_{x}, y=1, \cdot, N_{y}$ in a vector $\boldsymbol{f}$, we can write

$$
g_{i}=\mathcal{G}\left([\boldsymbol{H} \boldsymbol{f}]_{i}\right)+\epsilon_{i}, \quad i=1, \cdots, M \quad \text { or } \quad \boldsymbol{g}=\mathcal{G}(\boldsymbol{H} \boldsymbol{f})+\boldsymbol{\epsilon}
$$

where $\boldsymbol{H}$ is a matrix whose elements are related to exponential term in previous relation and $\boldsymbol{\epsilon}$ represent the errors.

\subsection{Bayesian MAP estimation}

To go further in detail, we assume the following:

$$
p(\boldsymbol{\epsilon})=\mathcal{N}\left(0, \sigma_{\epsilon}^{2} \boldsymbol{I}\right) \longrightarrow p(\boldsymbol{g} \mid \boldsymbol{f})=\mathcal{N}\left(\mathcal{G}(\boldsymbol{H} \boldsymbol{f}), \sigma_{\epsilon}^{2} \boldsymbol{I}\right)
$$

and

$$
p(\boldsymbol{f})=\mathcal{N}\left(0, \sigma_{f}^{2} \boldsymbol{\Sigma}_{f}\right) \quad \text { with } \quad \boldsymbol{\Sigma}_{f}=\left(\boldsymbol{D}^{t} \boldsymbol{D}\right)^{-1}
$$


where $\boldsymbol{D}$ is the matrix of first order finite differences.

Then, for the linear case $(\mathcal{G}(s)=s)$ it is easy to see that

$$
p(\boldsymbol{f} \mid \boldsymbol{g})=\mathcal{N}(\widehat{\boldsymbol{f}}, \widehat{\boldsymbol{\Sigma}}) \text { with } \widehat{\boldsymbol{\Sigma}}=\left(\boldsymbol{H}^{t} \boldsymbol{H}+\lambda \boldsymbol{I}\right)^{-1} \text { and } \widehat{\boldsymbol{f}}=\widehat{\boldsymbol{\Sigma}} \boldsymbol{H}^{t} \boldsymbol{g} \text { where } \lambda=\left(\sigma_{f}^{2} / \sigma_{\epsilon}^{2}\right)
$$

and that, $\widehat{\boldsymbol{f}}$ is also the MAP estimator which can be computed as an optimization problem:

$$
\widehat{\boldsymbol{f}}=\arg \max _{\boldsymbol{f}}\{p(\boldsymbol{f} \mid \boldsymbol{g})\}=\arg \min _{\boldsymbol{f}}\left\{J(\boldsymbol{f})=\|\boldsymbol{g}-\boldsymbol{H} \boldsymbol{f}\|^{2}+\lambda\|\boldsymbol{D} \boldsymbol{f}\|^{2}\right\}
$$

This can then be compared to the quadratic regularization.

\subsection{Mixture of Gaussian model for compact homogeneous regions modeling}

Now, we consider the case where we want a reconstructed image with homogeneous regions. This can be modeled by introducing a new variable $\boldsymbol{z}=\left[z_{1}, \cdots, z_{N}\right]$ where each $z_{j}$ takes a discrete value $k$ corresponding to the index of the region. Then defining the following notations

$$
R_{k}=\left\{j: z_{j}=k\right\}, \quad\left|R_{k}\right|=n_{k}, \text { and } \boldsymbol{f}_{k}=\left\{f_{j}: z_{j}=k\right\}
$$

and assuming that all the pixels with the same value $z_{j}=k$ are inside a homogeneous region with mean value $m_{k}$ and dispersions $\sigma_{k}$, we can write

$$
\begin{aligned}
p\left(\boldsymbol{f}_{k}\right)=\mathcal{N}\left(m_{k} 1, \sigma_{k}^{2} \boldsymbol{I}_{k}\right) & =\left(2 \pi \sigma_{k}^{2}\right)^{-n_{k} / 2} \exp \left\{-\frac{1}{2 \sigma_{k}^{2}} \sum_{j}\left(f_{j}-m_{k}\right)^{2}\right\} \\
& =\left(2 \pi \sigma_{k}^{2}\right)^{-n_{k} / 2} \exp \left\{-\frac{1}{2 \sigma_{k}^{2}}\left\|\boldsymbol{f}_{k}-m_{k} 1_{k}\right\|^{2}\right\}
\end{aligned}
$$

and

$$
p(\boldsymbol{f})=\prod_{k} \mathcal{N}\left(m_{k} 1, \sigma_{k}^{2} \boldsymbol{I}_{k}\right)=\prod_{k}\left(2 \pi \sigma_{k}^{2}\right)^{-n_{k} / 2} \exp \left\{-\frac{1}{2 \sigma_{k}^{2}}\left\|\boldsymbol{f}_{k}-m_{k} 1_{k}\right\|^{2}\right\}
$$

where $1_{k}$ is a vector of $k$ elements all equal to one. Thus, with this prior modeling, we have

$$
\widehat{\boldsymbol{f}}=\arg \max _{\boldsymbol{f}}\{p(\boldsymbol{f} \mid \boldsymbol{g})\}=\arg \min _{\boldsymbol{f}}\left\{J(\boldsymbol{f})=\|\boldsymbol{g}-\boldsymbol{H} \boldsymbol{f}\|^{2}+\sum_{k} \lambda_{k}\left\|\boldsymbol{f}_{k}-m_{k} 1_{k}\right\|^{2}\right\}
$$

with $\lambda_{k}=\left(\sigma_{k}^{2} / \sigma_{\epsilon}^{2}\right)$. However, note that these expressions depend on the partitions $R_{k}=\left\{j: z_{j}=k\right\}$ and thus on the labels $\boldsymbol{z}$ (classification and segmentation).

To include more explicitly this classification and segmentation, we write explicitly this dependence:

$$
p\left(f_{j} \mid z_{j}=k\right)=\mathcal{N}\left(m_{k}, \sigma_{k}^{2}\right)
$$

From here, we can go in three directions:

- Assuming $z_{j}$ iid with $P\left(z_{j}=k\right)=p_{k}$. Then

$$
P\left(z_{j}=k \mid f_{j}\right)=\frac{p\left(f_{j} \mid z_{j}=k\right) P\left(z_{j}=k\right)}{p\left(f_{j}\right)}=\frac{1}{p\left(f_{j}\right)} p_{k}\left(2 \pi \sigma_{k}^{2}\right)^{-1 / 2} \exp \left\{-\frac{1}{2 \sigma_{k}^{2}}\left(f_{j}-m_{k}\right)^{2}\right\}
$$

with

$$
p\left(f_{j}\right)=\sum_{k} p_{k}\left(2 \pi \sigma_{k}^{2}\right)^{-1 / 2} \exp \left\{-\frac{1}{2 \sigma_{k}^{2}}\left(f_{j}-m_{k}\right)^{2}\right\}
$$


We have then a tool to compute this posterior probability and define an estimator for it. For example the MAP estimate can be computed through a thresholding. Assuming the $m_{k}$ are ordered, i.e., $m_{1}<m_{2}<$ $\cdots<m_{K}$ we can define the thresholds $\left[s_{1}, \cdots, s_{K-1}\right]$ such that

$$
P\left(z_{j}=k \mid f_{j}=s_{k}\right)=P\left(z_{j}=k+1 \mid f_{j}=s_{k}\right), \quad k=1, \cdots, K-1
$$

i.e., the solutions of

$$
p_{k}\left(2 \pi \sigma_{k}^{2}\right)^{-1 / 2} \exp \left\{-\frac{1}{2 \sigma_{k}^{2}}\left(s_{k}-m_{k}\right)^{2}\right\}=p_{k+1}\left(2 \pi \sigma_{k+1}^{2}\right)^{-1 / 2} \exp \left\{-\frac{1}{2 \sigma_{k+1}^{2}}\left(s_{k}-m_{k+1}\right)^{2}\right\}
$$

and

$$
\widehat{z}_{j}=\left\{\begin{array}{ccc}
1 & \text { if } & f_{j} \leq s_{1} \\
k & \text { if } & s_{k}<f_{j} \leq s_{k+1}, \quad 2 \leq k \leq K-1 \\
K & \text { if } & s_{K-1}<f_{j}
\end{array}\right.
$$

The following figure shows plots of $p_{k} p\left(f_{j} \mid z_{j}=k\right), p\left(f_{j}\right)=\sum_{k} p_{k} p\left(f_{j} \mid z_{j}=k\right)$, and $P\left(z_{j}=k \mid f_{j}\right)$ as a function of $f_{j}$ for the particular case of $K=3, \sigma_{1}=.3, \sigma_{2}=.4, \sigma_{3}=.5$ and $p_{1}=p_{2}=p_{3}=1 / 3$. Note that, we always must have $\sum_{k} P\left(z_{j}=k \mid f_{j}\right)=1$.
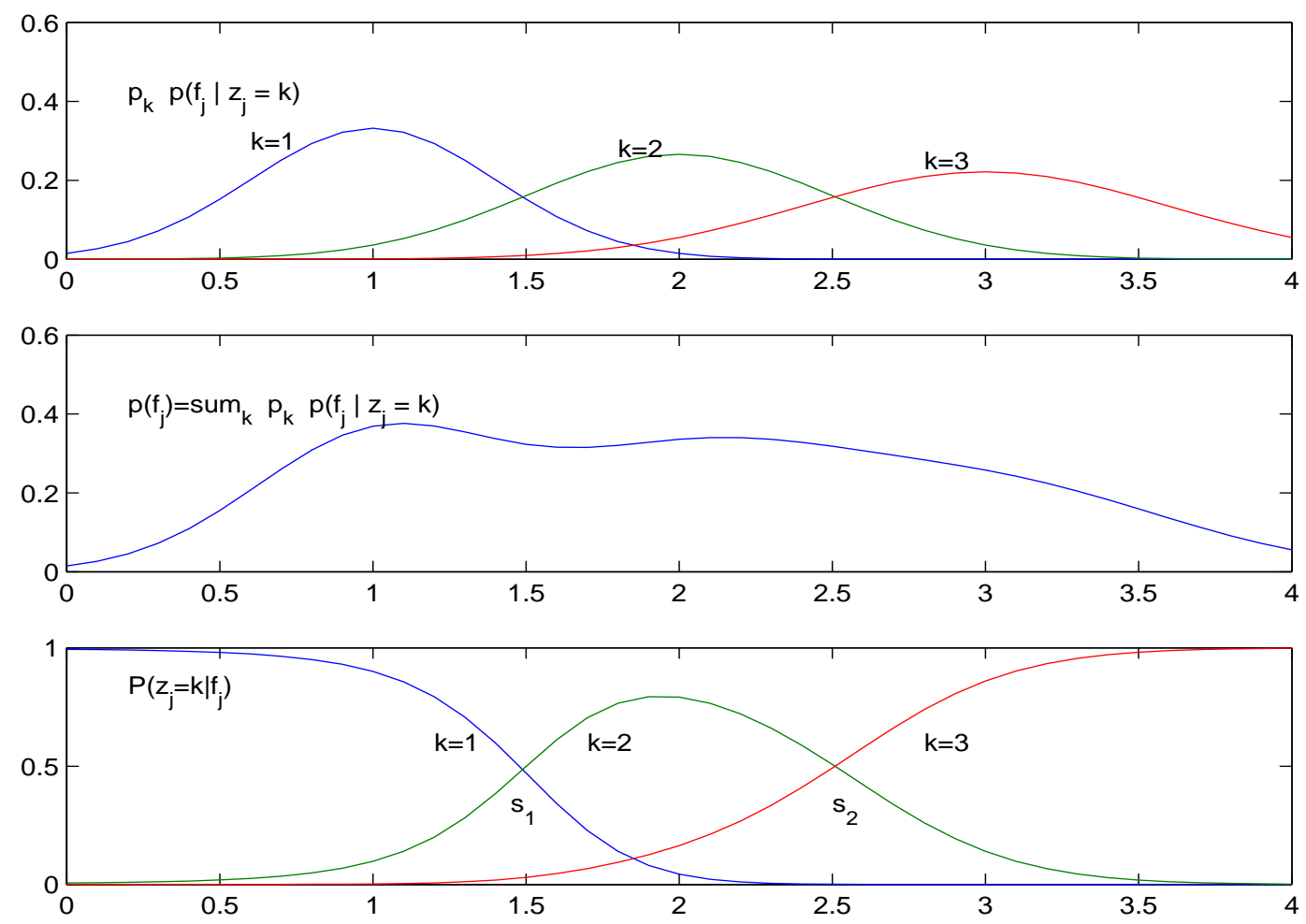

Figure 3. Likelihoods and posterior probabilities for classification: Weighted likelihoods $p_{k} p\left(f_{j} \mid z_{j}=k\right), k=$ $1,2,3$, the evidence $p\left(f_{j}\right)$ (middle) and posterior probabilities $P\left(z_{j}=k \mid f_{j}\right), k=1,2,3$ as functions of $f_{j}$. The two thresholds $s_{1}$ and $s_{2}$ are respectively solutions of $P\left(z_{j}=1 \mid f_{j}\right)=P\left(z_{j}=2 \mid f_{j}\right)$ and $P\left(z_{j}=2 \mid f_{j}\right)=P\left(z_{j}=3 \mid f_{j}\right)$.

Note that the thresholds $s_{k}$ are solutions of

$$
\ln p_{k}-\ln \sigma_{k}-\frac{1}{2 \sigma_{k}^{2}}\left(s_{k}-m_{k}\right)^{2}=\ln p_{k+1}-\ln \sigma_{k+1}-\frac{1}{2 \sigma_{k+1}^{2}}\left(s_{k}-m_{k+1}\right)^{2}
$$


or, equivalently, those of the second order polynomials

$$
\ln \frac{p_{k}}{p_{k+1}}-\ln \frac{\sigma_{k}}{\sigma_{k+1}}-\frac{1}{2 \sigma_{k}^{2}}\left(s_{k}-m_{k}\right)^{2}+\frac{1}{2 \sigma_{k+1}^{2}}\left(s_{k}-m_{k+1}\right)^{2}=0
$$

where only the solutions $m_{k}<s_{k}<m_{k+1}$ are valid.

Note also that the relation between this approach and the Level set approach for inverse problems for the case $K=2$. In this case, $\boldsymbol{f}$ plays the role of the Level set $\phi$ function with the difference that, in Level set approach the threshold is fixed. Indeed, this approach can be seen as an extension of the Level set approach to $K \geq 2$, which we call it Multi-Level set approach.

- Assuming a Markovian model for $z_{j}$ with $P\left(z_{j}=k \mid z_{j-1}=l\right)=p_{k l}$ with $\sum_{k} p_{k l}=1$. A simple homogeneous model is to choose $p_{k k}=\alpha_{k}$ and $p_{k l}=p_{l k}=\left(1-\alpha_{k}\right) /(K-1)$ which results to a vector $\boldsymbol{z}$ where each sub vector $\boldsymbol{z}_{k}$ is an exchangeable random vector. The parameters $\alpha_{k}$ fixes the mean size of the regions. With this modeling We have:

$$
\begin{aligned}
P\left(z_{j}=k \mid f_{j}, z_{j-1}=l\right) & =\frac{p\left(f_{j} \mid z_{j}=k\right) P\left(z_{j}=k \mid z_{j-1}=l\right)}{p\left(f_{j} \mid z_{j-1}=l\right)} \\
& =\frac{1}{p\left(f_{j} \mid z_{j-1}=l\right)} p_{k l}\left(2 \pi \sigma_{k}^{2}\right)^{-1 / 2} \exp \left\{-\frac{1}{2 \sigma_{k}^{2}}\left(f_{j}-m_{k}\right)^{2}\right\}
\end{aligned}
$$

with

$$
p\left(f_{j} \mid z_{j-1}=l\right)=\sum_{k} p_{k l}\left(2 \pi \sigma_{k}^{2}\right)^{-1 / 2} \exp \left\{-\frac{1}{2 \sigma_{k}^{2}}\left(f_{j}-m_{k}\right)^{2}\right\}
$$

We note that this time the threshold value to decide for $z_{j}=k$ against $z_{j}=k+1$, i.e.,

$$
P\left(z_{j}=k \mid f_{j}=s_{k}, z_{j-1}=l\right)=P\left(z_{j}=k+1 \mid f_{j}=s_{k}, z_{j-1}=l\right), \quad k=1, \cdots, K-1, l=k+1, \cdots, K
$$

or

$$
\ln \frac{p_{k, l}}{p_{k+1, l}}-\ln \frac{\sigma_{k}}{\sigma_{k+1}}-\frac{1}{2 \sigma_{k}^{2}}\left(s_{k}-m_{k}\right)^{2}+\frac{1}{2 \sigma_{k+1}^{2}}\left(s_{k}-m_{k+1}\right)^{2}=0, \quad k=1, \cdots, K-1, l=k+1, \cdots, K
$$

depend on the value of $z_{j-1}$, meaning that different values for pixels inside and pixels on the borders of the different regions. This is because, when we are inside a region, $z_{j}$ and $z_{j-1}$ take the same value $k$, but when we are on the borders of the regions they take different $k \neq l$ values. In general, a set of thresholds have to be computed. However, if we choose $p_{k k}=\alpha_{k}$ and $p_{k l}=p_{l k}=\left(1-\alpha_{k}\right) /(K-1)$, then we need to compute only the thresholds $s_{k k}, k=1, \cdots, K-1$ (for pixels inside the regions) and $s_{k l}, l \neq k$ (for pixels on the border of the regions).

In $2 \mathrm{D}$ case however, the situation is more complex because a pixel has more than one neighbor and the practical computation of these thresholds become more tricky.

- Another way to enforce the homogeneity of the pixel values inside each region is to put a Markovian structure between them. This can be done very easily by introducing a non diagonal covariance matrix in modeling $p\left(\boldsymbol{f}_{k}\right)$ :

$$
p\left(\boldsymbol{f}_{k}\right)=\mathcal{N}\left(m_{k} 1_{k}, \sigma_{k}^{2}\left(\boldsymbol{D}^{t} \boldsymbol{D}\right)^{-1}\right)
$$

with $\boldsymbol{D}$ a first order finite difference matrix. This can also be written differently through

$$
p\left(f_{j} \mid z_{j}=k, z_{j-1}=l\right)= \begin{cases}\mathcal{N}\left(f_{j-1}, \sigma_{k}^{2}\right) & k=l \\ \mathcal{N}\left(m_{k}, \sigma_{k}^{2}\right) & k \neq l\end{cases}
$$

meaning that $p\left(f_{j} \mid z_{j}=k\right)=\mathcal{N}\left(f_{j-1}, \sigma_{k}^{2}\right)$ for those pixels inside the regions (first order Markovian) and $p\left(f_{j} \mid z_{j}=k\right)=\mathcal{N}\left(m_{k}, \sigma_{k}^{2}\right)$ for those pixels on the borders of the regions. 
With this modeling, we can write:

$$
\begin{aligned}
P\left(z_{j}=k \mid f_{j}, z_{j-1}=l\right) & =\frac{p\left(f_{j} \mid z_{j}=k, z_{j-1}=l\right) P\left(z_{j}=k \mid z_{j-1}=l\right)}{p\left(f_{j} \mid z_{j-1}=l\right)} \\
& =\frac{1}{p\left(f_{j} \mid z_{j-1}=l\right)} p_{k l}\left(2 \pi \sigma_{k}^{2}\right)^{-1 / 2} \exp \left\{-\frac{1}{2 \sigma_{k}^{2}}\left(f_{j}-m_{k}\right)^{2}\right\}
\end{aligned}
$$

and

$$
\begin{aligned}
P\left(z_{j}=k \mid f_{j}, z_{j-1}=k\right) & =\frac{p\left(f_{j} \mid z_{j}=k, z_{j-1}=k\right) P\left(z_{j}=k \mid z_{j-1}=k\right)}{p\left(f_{j} \mid z_{j-1}=k\right)} \\
& =\frac{1}{p\left(f_{j} \mid z_{j-1}=k\right)} p_{k k}\left(2 \pi \sigma_{k}^{2}\right)^{-1 / 2} \exp \left\{-\frac{1}{2 \sigma_{k}^{2}}\left(f_{j}-f_{j-1}\right)^{2}\right\}
\end{aligned}
$$

In $1 \mathrm{D}$ case, we have again to consider two cases and two sets of thresholds have to be computed:

$$
\left\{\begin{array}{l}
P\left(z_{j}=k \mid f_{j}=s_{k}, z_{j-1}=k\right)=P\left(z_{j}=k+1 \mid f_{j}=s_{k}, z_{j-1}=k\right), \quad k=1, \cdots, K-1, \\
P\left(z_{j}=k \mid f_{j}=s_{k}, z_{j-1}=l\right)=P\left(z_{j}=k+1 \mid f_{j}=s_{k}, z_{j-1}=l\right), \quad k \neq l
\end{array}\right.
$$

In $2 \mathrm{D}$ case, the situation is easier than the previous case, because it is possible to replace $f_{j-1}$ by the mean value of the neighboring pixels (mean field approximation).

\subsection{General estimation algorithm}

In all these cases, the overall algorithm is the following:

1. Initial computation of $\widehat{\boldsymbol{f}}$ by optimizing $\quad J(\boldsymbol{f})=\|\boldsymbol{g}-\mathcal{G}(\boldsymbol{H} \boldsymbol{f})\|^{2}+\lambda\|\boldsymbol{f}\|^{2}$.

2. Initial setting of $s_{k}$, then initial determination of $R_{k}$ and initial computation of $m_{k}, \sigma_{k}^{2}$ and $p_{k}$ or $p_{k l}$ :

$$
\begin{aligned}
& n_{k}=\text { number of pixels in region } k \\
& m_{k}=\frac{1}{n_{k}} \sum_{j} f_{k_{j}}, \quad \sigma_{k}^{2}=\frac{1}{n_{k}} \sum_{j}\left(f_{k_{j}}-m_{k}\right)^{2}, \\
& p_{k}=n_{k} / n, \quad p_{k, l}=n_{k l} / \sum_{k} n_{k l}
\end{aligned}
$$

where $n$ is the total number of pixels and $n_{k l}$ the number of pixels in the common border of regions $l$ and $k$.

3. Computation of a new $\widehat{\boldsymbol{f}}$ by optimizing

$$
J(\boldsymbol{f})=\|\boldsymbol{g}-\mathcal{G}(\boldsymbol{H} \boldsymbol{f})\|^{2}+\sum_{k} \lambda_{k}\left\|\boldsymbol{f}-m_{k} 1_{k}\right\|^{2}
$$

or

$$
J(\boldsymbol{f})=\|\boldsymbol{g}-\mathcal{G}(\boldsymbol{H} \boldsymbol{f})\|^{2}+\sum_{k} \lambda_{k}\left[\sum_{j} q_{j}\left(f_{k_{j}}-m_{k}\right)^{2}+\sum_{j}\left(1-q_{j}\right)\left(f_{k_{j}}-f_{k_{j-1}}\right)^{2}\right]
$$

where $q_{j}=1$ for the pixels on the borders of the regions and $q_{j}=0$ for the pixels Inside the regions.

4. Classification and segmentation:

- Computation of $s_{k}$ or $s_{k l}$ which results to the determination of the regions $R_{k}$,

- Computation of $m_{k}, \sigma_{k}^{2}$ and $p_{k}$ or $p_{k l}$.

5. Return to 3. until some convergence condition is reached.

In the following, we show a set of results obtained in different domain using such methods. The comparisons are given with respect to the classical interpolation and inverse FT and with the Gerchberg-Papoulis or Gerchberg-Saxton type methods. 


\section{SIMULATION RESULTS}

To show some performances of the proposed method, we give here some simulation results which are summarized in The following figure. On this figure, a) shows an object simulating a hand with three kinds of materials, b) shows the external support of the object which is used as the prior knowledge, c) shows the support of the data in the Fourier domain and d) shows the data themselves. To simulates these data, we first computed the 2D FT of the object, added some noise (random samples from two independent Gaussians for real and imaginary parts) to represent the measurement noise. The signal to noise ratio for both real and imaginary parts was $20 \mathrm{~dB}$. Then, we considered two cases: i) a coherent imaging case where we assume to be able to measure the amplitude and the phase of the data on a given support and ii) the case of a non-coherent imaging where only the amplitude is measured. In both cases the data is assumed to be available on the circular support c) on this figure.

Then, using these data, we reconstructed the images either by a simple inverse FT when the phase is available e) or by the Gerchberg-Saxton algorithm when the phase is not available g). The figures f) and h) show the results obtained by the proposed method for those two cases.

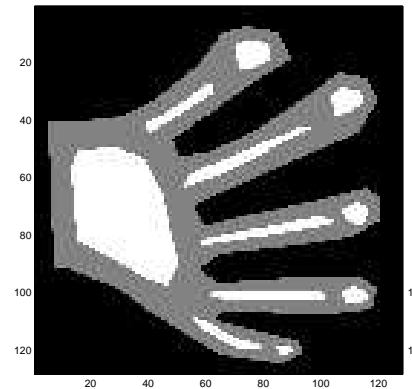

a)

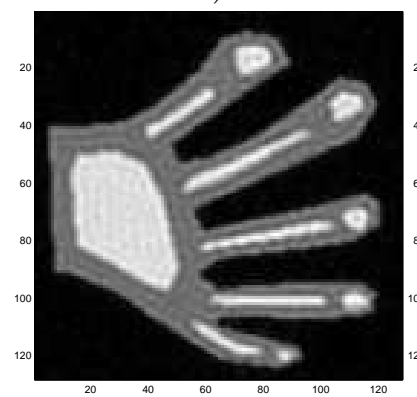

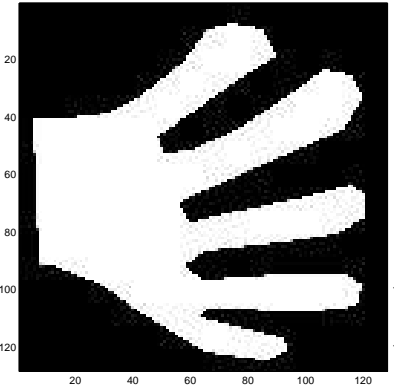

b)

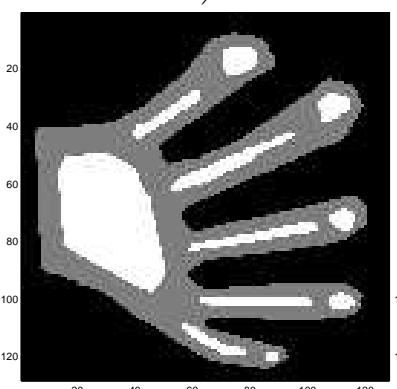

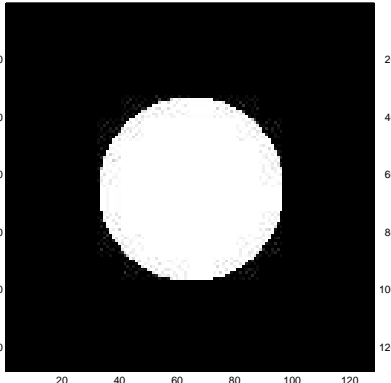

c)

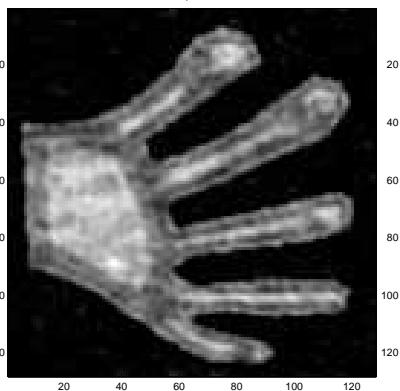

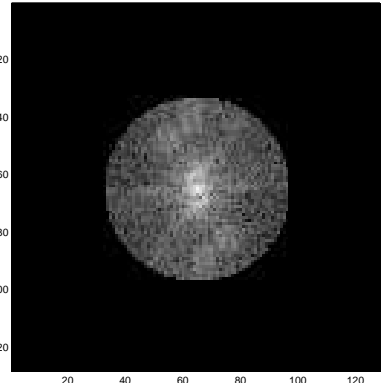

d)

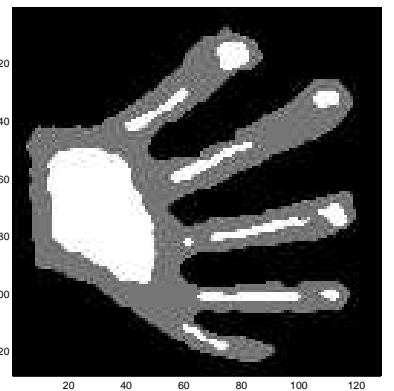

Figure 4. a) object, b) exact known support, c) support of the data, d) measured data, e) and f) Reconstruction results when the phase is measured: e) by IFT and by f) the proposed method, g) and h) Reconstruction results when the phase is not measured but we know the support of the object: g) by Gerchberg-Saxton h) by the proposed method.

\section{CONCLUSION}

In this paper we showed how an appropriate probabilistic modeling of the pixels distribution via a mixture of Gaussian in a Bayesian estimation framework can be used to obtain better reconstruction results in Fourier synthesis inverse problems of coherent and non coherent imaging. Even if the proposed method is obtained via the Bayesian estimation approach, the resulted algorithm can be considered as an extension of the Level-set methods. This is the reason for which we call the proposed algorithm Multi Level-set. 


\section{REFERENCES}

1. R. N. Bracewell, The Fourier Transform and its applications. International Ed.: MacGRAW-HILL Int. Ed., 1986.

2. A. C. Kak and M. Slaney, Principles of Computerized Tomographic Imaging. New York, NY: IEEE Press, 1987.

3. A. Mohammad-Djafari and G. Demoment, "Maximum entropy Fourier synthesis with application to diffraction tomography," Applied Optics, vol. 26, no. 10, pp. 1745-1754, 1987.

4. A. Mohammad-Djafari and G. Demoment, "Tomographie de diffraction et synthèse de Fourier à maximum d'entropie," Revue de Physique Appliqué, vol. 22, pp. 153-167, 1987.

5. A. Mohammad-Djafari and G. Demoment, "Maximum entropy reconstruction in X ray and diffraction tomography," IEEE Transactions on Medical Imaging, vol. MI-7, no. 4, pp. 345-354, 1988.

6. S. X. Pan and A. C. Kak, "A computational study of reconstruction algorithms for diffraction tomography: Interpolation versus filtered backpropagation," IEEE Transactions on Acoustics, Speech and Signal Processing, vol. ASSP-31, pp. 1262-1275, 1983.

7. A. Joisel and J.-C. Bolomey, "Rapid microwave imaging of living tissues," in Proceedings of SPIE's Int. Symp. on Medical Imaging, no. 3977, (San Diego, CA, USA), pp. 320-330, February 2000.

8. B. Duchêne, "Inversion of experimental data using linearized and binary specialized nonlinear inversion schemes," Inverse Problems, vol. 17, Special section (Saillard, M. and Belkebir, K. Ed.), pp. 1623-1634, December 2001.

9. R. Zorgati, B. Duchêne, D. Lesselier, and F. Pons, "Eddy current testing of anomalies in conductive materials, part I: Qualitative imaging via diffraction tomography techniques," IEEE Transactions on Magnetics, vol. 27, no. 6, pp. 4416-4437, 1991.

10. J. L. C. Sanz and T. S. Huang, "Some aspects of band-limited signal extrapolation: Models discrete approximations and noise," IEEE Transactions on Acoustics, Speech and Signal Processing, vol. ASSP31, pp. 1492-1501, 1983.

11. J. L. C. Sanz and T. S. Huang, "A unified approach to noniterative linear signal restoration," IEEE Transactions on Acoustics, Speech and Signal Processing, vol. ASSP-32, pp. 403-409, 1984.

12. J. L. C. Sanz, T. S. Huang, and Wu, "A annoteon iterative Fourier transform phase reconstruction from magnitude," IEEE Transactions on Acoustics, Speech and Signal Processing, vol. ASSP-32, December 1984.

13. A. Papoulis and M. S. Bertran, "Digital filtering and prolate functions," IEEE Transactions on Circuit Theory, vol. CT-19, pp. 674-681, 1972.

14. A. Papoulis, "A new algorithm in spectral analysis and band-limited extrapolation," IEEE Transactions on Circuits and Systems, vol. CS-22, pp. 735-742, September 1975.

15. R. W. Gerchberg, "Superresolution through error energy reduction," Optical Acta, vol. 21, no. 9, pp. 709720, 1974.

16. J. R. Fienup, "Phase retrieval algorithms: A comparison," Applied Optics, vol. 21, pp. 2758-2769, August 1982 .

17. M. C. Jones, "The discrete gerchberg algorithm," IEEE Transactions on Acoustics, Speech and Signal Processing, vol. ASSP-34, pp. 624-626, 1986.

18. J. Fienup, "Roconstruction of a complex-valued object from the modulus of its Fourier Transform using a support constraint," J. Opt. Soc. Am., vol. A 4, pp. 118-123, 1987. 